\title{
Optimal channel networks, landscape function and branched transport
}

\author{
FilipPo SANTAMBROGIO ${ }^{\dagger}$ \\ Scuola Normale Superiore, Piazza dei Cavalieri 7, 56126 Pisa, Italy
}

[Received 27 May 2006 and in revised form 19 June 2006]

\begin{abstract}
Starting from transportation models for branching structures, we define a function that represents the elevation of the landscape in a river basin. This function is already well-known in the geophysical community but it is only considered under a very strong discretization. We generalize it to the continuous case and study its properties, providing several applications.
\end{abstract}

\section{Introduction}

Lots of branching structures transporting different kind of fluids, such as road systems, communication networks, river basins, blood vessels, leaves and trees and so on, may be easily thought of as coming from a variational principle. They appear when transport costs encourage joint transportation. Recently these problems received a lot of attention by mathematicians. This paper mainly discusses some features wich are crucial in river basins applications, but addresses also applications to other fields.

\subsection{Branching transport models by Gilbert and Xia}

A mathematical formalization for the branching transport problems is very classical and has been performed first for atomic measures and then generalized. We briefly present here the problem introduced by Gilbert in [11] and [12], where it is presented as an extension of Steiner's minimal length problems. The main applications that Gilbert referred to were in the field of communication networks. Given two atomic probability measures $\mu=\sum_{i=1}^{m} a_{i} \delta_{x_{i}}$ and $v=\sum_{j=1}^{n} b_{j} \delta_{y_{j}}$, consider

$$
\left(P_{G}\right) \quad \min E(G):=\sum_{h} w_{h}^{\alpha} \mathcal{H}^{1}\left(e_{h}\right),
$$

where the infimum is among all weighted oriented graphs $G=\left(e_{h}, \hat{e}_{h}, w_{h}\right)_{h}$ (where $e_{h}$ are the edges, $\hat{e}_{h}$ represent their orientations and $w_{h}$ the weights) satisfying Kirchhoff's Law: at each vertex which is not one of the $x_{i}$ 's or $y_{j}$ 's the total incoming mass equals the outcoming, while at each $x_{i}$ we have

$$
a_{i}+\text { incoming mass }=\text { outcoming mass }
$$

and, conversely, at each $y_{j}$ we have

$$
\text { incoming mass }=\text { outcoming mass }+b_{j} .
$$

These conditions correspond exactly to the well-known Kirchhoff Law for electric circuits. The orientations $\hat{e}_{h}$ do not appear in the energy $E$ but appear in fact in Kirchhoff constraints. The

\footnotetext{
${ }^{\dagger}$ E-mail: f.santambrogio@sns.it
} 
exponent $\alpha$ is a fixed parameter $0<\alpha<1$ so that the function $t \mapsto t^{\alpha}$ is concave and subadditive. In this way larger links bringing the mass from $\mu$ to $v$ are preferred to several smaller links transporting the same total mass. It is not difficult to check that the energy of any finite graph may be improved if we remove cycles from the graph. In this way we can minimize among finite graphs which are actually trees. This implies a bound on the number of edges and hence ensures a suitable compactness which is enough to prove the existence of a minimizer.

More recently Xia, in [20], has proposed a new formalization leading to generalizations of this problem to arbitrary probability measures $\mu$ and $\nu$. In this case the interest of the author of [20] is to view this problem as an extension of Monge-Kantorovich optimal transport theory (see [19]). In fact Steiner and Monge's problems represent the limit cases $\alpha=0$ and $\alpha=1$, respectively.

\subsection{Landscape equilibrium and OCNs in geophysics}

It is interesting to see how people working in geophysics arrive at very similar problems in the study of river basins. There is a wide literature on this and a quite comprehensive reference is [17]. The specific subject dealt with by this paper is developed both in [17] and in [2] (this last paper being our main reference, but a short previous summary of these ideas can be found in [1] as well). While studying the configuration of a river basin, the main objects are two: the landscape elevation, which is a function $z$ giving the altitude of any point of the region we are considering, and a river network $N$, which is the datum of all the streams that concur to bring water (which falls on the region as rain) to a single point (where a lake is supposed to be present). A first link between both objects is the fact that at any point the direction followed by water is the direction of steepest descent of $z$. Hence, once we know $z$ we are able to deduce $N$ and to compute the multiplicity $\theta(x)$ at any point $x$, which is the quantity of water passing through $x$ while following the steepest descent lines of $z$. At first the interest is towards an evolution model, which allows $z$ and $N$ (and hence $\theta$ ) to depend on time as well. The evolution of $z$ is ruled by an erosion equation of the form

$$
\frac{\partial z}{\partial t}=-\theta|\nabla z|^{2}+c,
$$

where $\nabla z$ is the spatial gradient of $z$ and $c$ is a positive constant. The idea is that the erosion effect increases both with the quantity of water and with the slope. The constant $c$ is called uplift and takes care of the fact that all the material brought down by erosion is in the end uniformly redistributed from below in the whole region as a geomorphological effect. Equation (1.2) is in fact a simplified version of other more general evolution equations involving higher order terms. The following phenomenon concerning solutions of (1.2) can be empirically observed: approximately, up to a certain time scale both $z$ and $\theta$ (i.e. $N$ ) move, in a very strong erosional evolution; then, up to a larger time scale the network is almost constant, letting $\theta(x, t)=\theta(x)$ depend on the position only, and the landscape function evolves without changing its lines of maximal slope; finally, there is a much larger time scale such that $z$ approximately agrees with a landscape equilibrium, i.e. a stationary solution of 1.2 . We are interested in studying landscape equilibria. In this case the steepest descent condition, which we can read as " $\nabla z$ follows the direction of the network", is completed by a second one which we get by imposing $\partial z / \partial t=0$ in $[1.2)$. This leads to $|\nabla z|=c^{1 / 2} \theta^{-1 / 2}$ and this last condition is called the slope-discharge relation. It is explicitly suggested in [2] that in [1.2] one could change the exponents of $\theta$ and $|\nabla z|$ (preserving anyway the increasing behavior with respect to both variables), thus obtaining different slope-discharge relationships. In general we get $|\nabla z|=c \theta^{\alpha-1}$ and the physically interesting case is when the exponent $\alpha$ is very close to $1 / 2$. 
To find landscape equilibria a discretization is performed in [2] and a regular square grid is used. Functions defined on the pixels of the grid and vanishing at a given point $x_{0}$ representing the outlet are considered, as well as networks composed by edges of the grid, directed from every point to one of the neighbors.

- As we already mentioned, the conditions on the direction of the water allow reconstructing a network from a function. In fact, given a function $z$ with no local minima other than $x_{0}$, one can always follow the maximal slope paths of $z$.

- These are obtained by linking any point $x$ of the grid to a point which realizes the minimum of $z$ among the neighbors of $z$. Notice in particular that these paths are only composed by edges following the two main directions of the grid.

- In this way a network $N=N(z)$ can be deduced from $z$.

- On the other hand, the slope-discharge condition allows one to reconstruct a function from a network $N$, provided it is tree-shaped.

- In order to make this reconstruction, first compute the multiplicities of the points of the network: at a point $x$ its multiplicity $\theta(x)$ is the number of points which find $x$ on their way to the outlet (this works under the assumption that the quantity of rain falling down at any pixel is the same, i.e. rain falls uniformly on the grid). See also Figure 1., where the multiplicity of a point $x_{i}$ is computed as the number of points in the area $A_{i}$.

- Then set $z\left(x_{0}\right)=0$ and for any other point $x$ consider the only path on $N$ linking $x_{0}$ to $x$. Set $z(x)=\sum_{i} \theta\left(x_{i}\right)^{\alpha-1}$, where the $x_{i}$ 's are the points on the path. In Figure 1 the path linking $x_{0}$ to $x$ is shown.

- In this way we get a function $z=z(N)$.

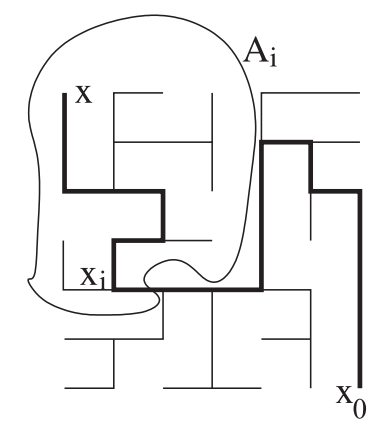

FIG. 1. The path from $x$ to $x_{0}$ and the multiplicity of $x_{i}$.

In general it will not be true that a function $z(N)$ has maximal slope in the direction of the network $N$. Finding a landscape equilibrium means exactly satisfying both conditions at a time, through a fixed point problem. The algorithm starts from a tree-shaped network $N$, builds the function $z(N)$, and then the new network $N^{\prime}=N(z(N))$. If $N^{\prime}=N$, then the landscape function $z=z(N)$ is a landscape equilibrium.

The important idea presented in [2] is the relation between landscape equilibria and Optimal Channel Networks (OCNs in literature, see for instance [18], [16] and [13]). An OCN is a network $N$ minimizing a certain dissipated energy. The dissipated energy in a system satisfying the slopedischarge relation is the total potential energy that water loses on the network. For each pixel we have a quantity of water $\theta$ which falls down towards the next pixel and its elevation decreases by 
a quantity which is proportional to $|\nabla z|$ and hence to $\theta^{\alpha-1}$. Hence, the total energy loss is given by $\sum_{i} \theta\left(x_{i}\right) \theta\left(x_{i}\right)^{\alpha-1}=\sum_{i} \theta\left(x_{i}\right)^{\alpha}$. It is clear that this energy is the same as in (1.1) (no length of segments is involved because in a regular grid they all have the same, given, length). What is proven in [2] is that, if $N$ is an OCN minimizing this energy, then the landscape function $z=z(N)$ reconstructed from it is in fact an equilibrium. This actually means that not only is the slope of $z$ in the direction of the network given by $\theta^{\alpha-1}$, which is true by construction, but also this direction is the direction of maximal slope.

Notice that the problems studied in [2] and in the other papers on the subject have undergone a very strong discretization. In fact, they correspond to solving $\left(P_{G}\right)$ where $\mu$ is a discretization on a regular grid of the Lebesgue measure and $v=\delta_{x_{0}}$, but with the extra constraint that only edges $e_{h}$ which are given with the grid are allowed. Compared to continuous models there is a loss of rotational invariance, a fixed scale effect due to the mesh, and several questions concerning the river basin may lose their meaning (for instance questions about the interfaces between two separated parts of the basin and points where the water takes two different directions, or most regularity issues). On the other hand, a continuous counterpart for the landscape function could not be simply a regular solution of (1.2) or of its statical version, as for $C^{1}$ functions steepest descent curves are well-defined, but they never merge and therefore do not give rise to a positive multiplicity $\theta$ (except for the case $d=1$, see [2]).

\subsection{A landscape function appearing for derivation purposes}

We will briefly see here another aspect of branching transport problems such as $\left(P_{G}\right)$ where a function similar to the landscape function appears.

Definition 1 We define the irrigation cost of a finite atomic measure $\mu \in \mathcal{P}(\Omega)$ to be the minimum of problem $\left(P_{G}\right)$ for $v=\delta_{0}$. This quantity is denoted by $X_{\alpha}(\mu)$.

A variational analysis of this functional yields the following.

THEOREM 1.1 Suppose that $\mu=\sum_{i=1}^{m} a_{i} \delta_{x_{i}}$ with $a_{i}>0$ (so that the finite set $K=\left\{x_{i}: i=\right.$ $1, \ldots, n\}$ is actually the support of $\mu$ ) and that $\mu_{1}$ is another probability measure concentrated on $K$ with $\mu_{1}=\sum_{i=1}^{m} b_{i} \delta_{x_{i}}$. Then

$$
X_{\alpha}\left(\mu_{1}\right) \leqslant X_{\alpha}(\mu)+\alpha \sum_{i=1}^{m} z\left(x_{i}\right)\left(b_{i}-a_{i}\right),
$$

where the function $z$ is defined in this way: take an optimal graph $G$ for problem $\left(P_{G}\right)$ for the measures $\mu$ and $\delta_{0}$; this graph is a tree; for any $x_{i}$ define

$$
z\left(x_{i}\right)=\sum_{h \in H(i)} w_{h}^{\alpha-1} \mathcal{H}^{1}\left(e_{h}\right),
$$

where $H(i)$ denotes the set of the indices of the edges of the unique path from 0 to $x_{i}$.

Proof. We will build a new oriented graph which is acceptable for problem $\left(P_{G}\right)$ when irrigating $\mu_{1}$ starting from $\delta_{0}$. This graph will be built by using the same edges $\left(e_{h}\right)_{h}$ as in $G$ but changing the weights $w_{h}$. We define the new weights $w_{h}^{\prime}$ by

$$
w_{h}^{\prime}=w_{h}+\sum_{i: h \in H(i)}\left(b_{i}-a_{i}\right)
$$


It is easy to check that this new graph satisfies the constraints, and so we get

$$
X_{\alpha}\left(\mu_{1}\right) \leqslant \sum_{h}\left(w_{h}^{\prime}\right)^{\alpha} \mathcal{H}^{1}\left(e_{h}\right) \leqslant X_{\alpha}(\mu)+\alpha \sum_{h} w_{h}^{\alpha-1} \sum_{i: h \in H(i)}\left(b_{i}-a_{i}\right),
$$

where the last inequality is obtained by concavity of $t \mapsto t^{\alpha}$. By changing the order in performing the sums we easily get the assertion.

REMARK 1 The link between this function $z$ and the one used in geophysics is straightforward: to compute a value $z(x)$, what we do in fact is integrating the multiplicity of the graph along the river from 0 up to $x$. See Figure 2 and compare with Figure 1 in this case there are in general many more degrees of freedom, the multiplicity of the represented point $x_{i}$ is the total mass of the region $A_{i}$ and the geometry of points and edges is not prescribed.

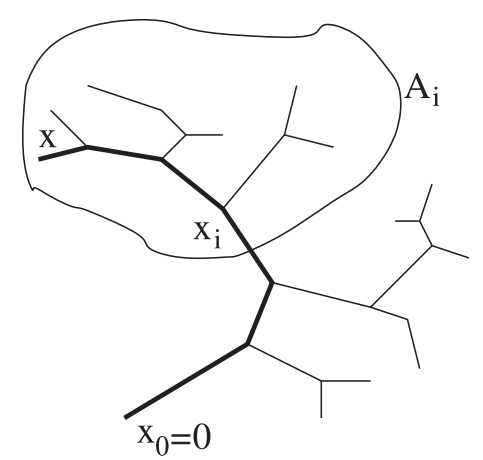

FIG. 2. The path from $x$ to $x_{0}$ and the multiplicity of $x_{i}$.

REMARK 2 As a consequence of Theorem 1.1 if we set $\mu_{\varepsilon}=\mu+\varepsilon\left(\mu_{1}-\mu\right)$, we get

$$
\limsup _{\varepsilon \rightarrow 0^{+}} \frac{X_{\alpha}\left(\mu_{\varepsilon}\right)-X_{\alpha}(\mu)}{\varepsilon} \leqslant \int z \mathrm{~d}\left(\mu_{1}-\mu\right) .
$$

This inequality gives information on the derivative of the functional $X_{\alpha}$ and this fact is very useful in variational problems of the following kind:

$$
\left(P_{+}\right) \quad \min X_{\alpha}(\mu)+F(\mu),
$$

where $F$ may be any functional whose derivative is known. We will show later an example and briefly explain the interest of these problems.

REMARK 3 Theorem 1.1 has been established under no constraints on the direction of the edges, i.e. in the setting of problem $\left(P_{G}\right)$. It is easy to reproduce them in the case of grid-constrained OCNs, as in the proof there is no need to change the edges of the graph. Hence this result is also valid in the setting of [2].

\subsection{Goals of the paper}

The main goal of this paper is to define a landscape function in the continuous case and analyze its properties. We will use the recent developments about these irrigation problems concerning arbitrary probability measures (and not only atomic ones) that we mentioned before. We will consider the 
irrigation of an arbitrary measure on a domain $\Omega$ starting from a single source $\delta_{0}$. Here the main problem is that the optimal structures which arise are not necessarily trees in the sense that there may be points which are reached by several curves. We will anyway propose a landscape function $z$ and check that it is well-defined. Then we will prove that it shares all the properties that we had in the discrete case, in particular at a point $x_{0}$ of the irrigation network it has maximal slope in the direction of the network itself and this slope is given by $\theta^{\alpha-1}$, where $\theta$ is the multiplicity of the network at $x_{0}$. Moreover we will prove that even in the continuous case an inequality on the derivative of the energy $X_{\alpha}$ involving the landscape function is available, and finally we will give some continuity and semicontinuity results.

Generalizing the concept of landscape function to the irrigation of arbitrary probability measures has not only variational applications. In river basin applications, in fact, it is natural to consider directly a configuration where the starting measure is the Lebesgue measure instead of considering a grid discretization. Moreover, getting rid of the discretization will also add isotropy and other features to the models in [2] and [17].

The main results leading to the definition of a landscape function and to the study of its properties will be presented from Section 3 on. Section 2 is in fact devoted to a short summary of the different models and of the main features that will be used later.

\section{Branched transport structures: models and tools}

Recently, several models have been developed to study branching transport structures. At first sight they are very different, but many equivalences can be proved. On one hand there are the Eulerian approaches, first by Gilbert and then by Xia, and on the other there are Lagrangian, time-dependent approaches mainly presented in [15] and [4]. The Eulerian models are the most similar to the one used by the geophysical community, and the generalization by Xia will be explained here a little more. The Lagrangian ones, on the other hand, are the most useful to deal with a possible landscape function thanks to the form of the functionals involved.

\subsection{Relaxed minimization by Xia and its minimal value}

Let us briefly see how Xia extended the Gilbert approach. The key point is that the constraint on the incoming and outcoming masses at each vertex (Kirchhoff Law) may be easily written as $\nabla \cdot \lambda_{G}=$ $\mu-v$, where $\lambda_{G}=\sum_{h} w_{h}\left[\left[e_{h}\right]\right]$ is a vector measure ([[e]] being the integration measure on the segment $e$ following its direction: a measure which is absolutely continuous with respect to $\mathcal{H}^{1}$ with density $\hat{e}$ on $e$ and 0 elsewhere). These considerations led Xia in [20] to extend the problem by relaxation to generic probabilities $\mu$ and $\nu$. The problem becomes

$$
\left(P_{X}\right) \quad \min \bar{E}(\lambda): \nabla \cdot \lambda=\mu-v
$$

where $\bar{E}(\lambda):=\inf \liminf _{n} E\left(\lambda_{G_{n}}\right)$ with the infimum taken over all possible sequences $\left(G_{n}\right)_{n}$ of finite graphs such that the corresponding vector measures $\lambda_{G_{n}}$ converge to $\lambda$.

One can prove that, when $\mu$ and $v$ are both actually atomic measures, we retrieve the problem by Gilbert. This can be done by means of necessary optimality conditions: if we minimize Xia's functional over vector measures, we can prove that a minimizer must necessarily be a finite graph (see [21] and [5]). Hence Xia's formulation is an extension of Gilbert's.

The minimum value of $\left(P_{X}\right)$, which obviously depends on $\mu$ and $\nu$, will be denoted by $d_{\alpha}(\mu, v)$. It is very important to understand when this minimal value is finite. It is proven in [20] that, if $\alpha$ 
is sufficiently close to 1 , namely $\alpha>1-1 / d$, then this minimum is finite for any pair $(\mu, v)$. Moreover, the following uniform estimate (see [20]) holds:

$$
d_{\alpha}(\mu, \nu) \leqslant C_{\alpha, d} \operatorname{diam}(\Omega) .
$$

To deal with local perturbations, it is not difficult to deduce a sharper estimate, namely

$$
d_{\alpha}(\mu, v) \leqslant C_{\alpha, d} \delta^{\alpha} \operatorname{diam}(\omega),
$$

whenever $\mu-v=\delta\left(\mu^{\prime}-v^{\prime}\right)$ and $\mu^{\prime}$ and $v^{\prime}$ are probability measures on $\omega \subset \Omega$. When $\alpha$ is below the threshold $1-1 / d$ there are pairs of measures which are not linkable by a finite energy configuration. The possibility of reaching a certain measure by finite energy is somehow linked to its "dimension" (see [8]).

DEFinition 2 For any measure $\mu \in \mathcal{P}(\Omega)$ we set $X_{\alpha}(\mu)=d_{\alpha}\left(\mu, \delta_{0}\right)$. This is an extension of Definition 1. Moreover, a measure $\mu$ is called $\alpha$-irrigable if $X_{\alpha}(\mu)<+\infty$.

In [20] it is proven that, for $\alpha>1-1 / d$, the quantity $d_{\alpha}$ defines a new distance over the space $\mathcal{P}(\Omega)$ of probability measures, which induces the weak topology. Both the continuity with respect to this topology and the triangle inequality will be used in the following.

\subsection{Patterns and traffic plan models}

This subsection is an informal summary of the models in [15] and [4] and their properties. The approach and terminology have been sometimes simplified in view of the aim of this paper.

Let $\Omega$ be a fixed compact domain in $\mathbb{R}^{d}$. Denote by $\Gamma$ the set of 1-Lipschitz curves $\gamma$ : $[0,+\infty[\rightarrow \Omega$ that are eventually constant. This means that, if we define the stopping time of a curve $\gamma$ by

$$
\sigma(\gamma)=\inf \{s: \gamma \text { is constant on }[s,+\infty[\},
$$

these are curves with $\sigma(\gamma)<+\infty$. Denote by $\Gamma_{\text {arc }}$ the set of those curves in $\Gamma$ which are parametrized by arc length, and by $\Gamma_{\text {inj }}$ the set of curves in $\Gamma$ which are injective on $[0, \sigma(\gamma)[$. We will often identify a curve with its image, writing $\gamma$ instead of $\gamma([0, \sigma(\gamma)])=\gamma([0,+\infty[)$.

Given a probability measure $\eta$ on the space $\Gamma$, for any point $x \in \mathbb{R}^{d}$ the $\eta$-multiplicity of $x$ is defined by

$$
[x]_{\eta}:=\eta\{\gamma \in \Gamma: x \in \gamma([0, \sigma(\gamma)])\} .
$$

Then we can define

$$
Z_{\eta}(\gamma)=\int_{0}^{\sigma(\gamma)}[\gamma(t)]_{\eta}^{\alpha-1} \mathrm{~d} t \quad \text { and } \quad J(\eta)=\int_{\Gamma} Z_{\eta} \mathrm{d} \eta .
$$

Notice that, for simplicity, here $Z_{\eta}$ is defined without the term $\left|\gamma^{\prime}\right|(t)$ which appears in the original definition in [4]. It will be deduced later that minimizers are actually parametrized by arc length.

Finally, we consider the maps $\pi_{0}, \pi_{\infty}: \Gamma \rightarrow \Omega$ given by $\pi_{0}(\gamma)=\gamma(0)$ and $\pi_{\infty}(\gamma)=$ $\gamma(\sigma(\gamma))$. The two image measures $\left(\pi_{0}\right)_{\sharp} \eta$ and $\left(\pi_{\infty}\right)_{\sharp} \eta$, which belong to $\mathcal{P}(\Omega)$, will be called the starting measure and terminal measure of $\eta$, respectively. Following the terminology of [4] we define a traffic plan as a measure $\eta \in \mathcal{P}(\Gamma)$ such that $\int_{\Gamma} \sigma(\gamma) \eta(\mathrm{d} \gamma)<+\infty$. We will also call a traffic plan $\eta$ such that $\left(\pi_{0}\right)_{\sharp} \eta=\delta_{0}$ a pattern. In the case of a pattern the terminal measure will also be called the measure irrigated by $\eta$. 
The minimization problem proposed in [4] is

$$
\text { (P) } \min \left\{J(\eta): \eta \text { is a traffic plan, }\left(\pi_{\infty}\right)_{\sharp \eta}=\mu,\left(\pi_{0}\right)_{\sharp \eta}=\nu\right\},
$$

where $\mu$ and $v$ are given measures in $\mathcal{P}(\Omega)$. As $[\gamma(t)]_{\eta} \leqslant 1$, we have $Z_{\eta}(\gamma) \geqslant \sigma(\gamma)$. Hence it is straightforward that any $\eta$ such that $J(\eta)<+\infty$ is actually a traffic plan. A traffic plan $\eta$ which minimizes $J$ among the traffic plans with the same starting and terminal measures, with $J(\eta)<+\infty$, will be called an optimal traffic plan. In the case $v=\delta_{0}$ it will be called an optimal pattern.

A useful tool developed in [4] (see also [3]) is the following: if $\eta$ is concentrated on $\Gamma_{\mathrm{arc}} \cap \Gamma_{\mathrm{inj}}$ then the following remarkable formula holds:

$$
J(\eta)=\int_{\mathbb{R}^{d}}[x]_{\eta}^{\alpha} \mathcal{H}^{1}(\mathrm{~d} x) .
$$

This formula gives an evident link with Gilbert and Xia's models.

\subsection{Useful tools: optimality conditions for $(P)$}

Before presenting some consequences of optimality, let us deal with some concepts from [15] which are crucial in the case $v=\delta_{0}$.

For any $t \geqslant 0$ consider an equivalence relation on $\Gamma$ given by "the curves $\gamma_{1}$ and $\gamma_{2}$ are in relation at time $t$ if they agree on the interval $[0, t]$ ", and denote the equivalence classes by $[\cdot]_{t}$, so that

$$
[\gamma]_{t}=\{\tilde{\gamma}: \tilde{\gamma}(s)=\gamma(s) \text { for any } s \leqslant t\} .
$$

For notational simplicity, set $|\gamma|_{t, \eta}:=\eta\left([\gamma]_{t}\right)$.

Definition 3 Given $\eta \in \mathcal{P}(\Gamma)$, a curve $\gamma \in \Gamma$ is said to be $\eta$-good if

$$
\int_{0}^{\sigma(\gamma)}|\gamma|_{t, \eta}^{\alpha-1} \mathrm{~d} t<+\infty
$$

Here are the most important results that can be found in [15], [4], [3], [5] and [14] or easily deduced from them.

(1) Problem $(P)$ admits a solution, provided the infimum is finite (i.e. there is at least a solution with finite energy).

(2) If $\eta$ is an optimal traffic plan, then $\eta$ is concentrated on $\Gamma_{\text {arc }} \cap \Gamma_{\text {inj. }}$. In particular, we may apply formula $(2.3)$ for $J$.

(3) Suppose that $\eta$ is an optimal traffic plan, that two curves $\gamma_{0}, \gamma_{1} \in \Gamma_{\text {arc }} \cap \Gamma_{\text {inj }}$ meet twice (i.e. $\gamma_{0}\left(s_{0}\right)=\gamma_{1}\left(s_{1}\right), \gamma_{0}\left(t_{0}\right)=\gamma_{1}\left(t_{1}\right)$ and $\left.s_{i} \neq t_{i}\right)$ and that $\left[\gamma_{0}(t)\right]_{\eta} \geqslant c>0$ for any $t \in$ $\left[s_{0}, t_{0}\right]$. Then either both curves coincide in the trajectory between the two common points or we have $\int_{s_{0}}^{t_{0}}\left[\gamma_{0}(t)\right]_{\eta}^{\alpha-1} \mathrm{~d} t<\int_{s_{1}}^{t_{1}}\left[\gamma_{1}(t)\right]_{\eta}^{\alpha-1} \mathrm{~d} t$. In particular two different curves with multiplicities bounded from below cannot part and then meet again.

(4) If $\eta$ is an optimal pattern (in particular $v=\delta_{0}$ ), then for $\eta$-a.e. curve $\gamma$ and a.e. $t<\sigma(\gamma)$ we have $[\gamma(t)]_{\eta}=\eta\left([\gamma]_{t}\right)$. Roughly speaking, this means that if all the mass starts from a common point then there is no parting-and-meeting-again-later (this is the single path property described in [5]). 
(5) As a consequence, any optimal pattern $\eta$ is concentrated on the set of $\eta$-good curves, and any $\eta$-good curve $\gamma$ belongs to $\Gamma_{\text {arc }} \cap \Gamma_{\text {inj }}$ and satisfies $[\gamma(t)]_{\eta}=\eta\left([\gamma]_{t}\right)$ for any $t<\sigma(\gamma)$.

(6) Last but not least, $\min (P)=d_{\alpha}(\mu, v)$, which means that the minima of the Lagrangian and of the Eulerian models coincide.

REMARK 4 Notice that an optimal traffic plan $\eta$ is concentrated on the set of $\eta$-good curves, but this does not mean that this set is linked to the support of $\eta$. In fact, any restriction of an $\eta$-good curve is itself an $\eta$-good curve and hence, for instance, in the discrete case, we have plenty of $\eta$-good curves but the support of $\eta$ is finite. In particular, the set of $\eta$-good curves may be very different from the set of fibers of a traffic plan that we find in [4] or [5] and does not depend on any parametrization, but it is more intrinsic.

\section{A general development formula}

In this section we will develop in a useful way the variation of the functional $J$ when passing from a traffic plan $\eta$ to a traffic plan $\eta^{\prime}$. Formula $(2.3)$ will be crucial.

THEOREM 3.1 Let $\eta$ and $\eta^{\prime}$ be probability measures on $\Gamma$ and $\Delta \eta=\eta^{\prime}-\eta$. Suppose that both $\eta$ and $\Delta \eta$ are concentrated on $\Gamma_{\operatorname{arc}} \cap \Gamma_{\text {inj }}$ and $\int_{\Gamma} Z_{\eta} \mathrm{d}|\Delta \eta|<+\infty$. Then

$$
J\left(\eta^{\prime}\right) \leqslant J(\eta)+\alpha \int_{\Gamma} Z_{\eta} \mathrm{d} \Delta \eta-\alpha(1-\alpha) \int_{\mathbb{R}^{d}}[x]_{\Delta \eta}^{2} \mathcal{H}^{1}(\mathrm{~d} x) .
$$

Proof. Set $S_{\eta}=\left\{x \in \mathbb{R}^{d}:[x]_{\eta}>0\right\}$ and analogously $S_{\eta^{\prime}}=\left\{x \in \mathbb{R}^{d}:[x]_{\eta^{\prime}}>0\right\}$. First we prove that under the assumptions of this theorem, $\mathcal{H}^{1}\left(S_{\eta^{\prime}} \backslash S_{\eta}\right)=0$. In fact, for any $x \in S_{\eta^{\prime}} \backslash S_{\eta}$ we have necessarily $[x]_{\eta}=0$ and $[x]_{\Delta \eta}>0$. Hence it is sufficient to prove that the integral of $[x]_{\Delta \eta}$ on this set vanishes to get the desired result. We have

$$
\int_{S_{\eta^{\prime}} \backslash S_{\eta}}[x]_{\Delta \eta} \mathcal{H}^{1}(\mathrm{~d} x)=\int_{S_{\eta^{\prime}} \backslash S_{\eta}} \mathcal{H}^{1}(\mathrm{~d} x) \int_{\Gamma} \Delta \eta(\mathrm{d} \gamma) I_{x \in \gamma}=\int_{\Gamma} \Delta \eta(\mathrm{d} \gamma) \mathcal{H}^{1}\left(\gamma \cap\left(S_{\eta^{\prime}} \backslash S_{\eta}\right)\right),
$$

where $I$ denotes the function whose value is 1 if the condition in the subscript is satisfied, 0 otherwise (indicator function). The second assumption of the theorem implies that for $\Delta \eta$-a.e. curve $\gamma$ the quantity $Z_{\eta}(\gamma)$ is finite, and hence $\gamma(t) \in S_{\eta}$ for a.e. $t$. Since $\gamma$ is 1-Lipschitz continuous, this yields $\mathcal{H}^{1}\left(\gamma \backslash S_{\eta}\right)=0$. Hence $\int_{S_{\eta^{\prime}} \backslash S_{\eta}}[x]_{\Delta \eta} \mathcal{H}^{1}(\mathrm{~d} x)=0$, which proves $\mathcal{H}^{1}\left(S_{\eta^{\prime}} \backslash S_{\eta}\right)=0$.

Now, as both $\eta$ and $\eta^{\prime}$ are concentrated on $\Gamma_{\text {arc }} \cap \Gamma_{\mathrm{inj}}$, to evaluate $J$ we can use the expression in 2.3 and get

$$
\begin{aligned}
J\left(\eta^{\prime}\right) & =\int_{S_{\eta}}\left([x]_{\eta}+[x]_{\Delta \eta}\right)^{\alpha} \mathcal{H}^{1}(\mathrm{~d} x) \\
& \leqslant J(\eta)+\alpha \int_{S_{\eta}}[x]_{\eta}^{\alpha-1}[x]_{\Delta \eta} \mathcal{H}^{1}(\mathrm{~d} x)-\alpha(1-\alpha) \int_{S_{\eta}}[x]_{\Delta \eta}^{2} \mathcal{H}^{1}(\mathrm{~d} x),
\end{aligned}
$$

where we have used the fact that $S_{\eta^{\prime}} \subset S_{\eta}$ up to $\mathcal{H}^{1}$-negligible sets and the concavity inequalities

$$
(t+s)^{\alpha} \leqslant t^{\alpha}+\alpha t^{\alpha-1} s-\alpha(1-\alpha)(\max \{t, t+s\})^{\alpha-2} s^{2} \leqslant t^{\alpha}+\alpha t^{\alpha-1} s-\alpha(1-\alpha) s^{2}
$$

(this last inequality being valid when both $t$ and $t+s$ belong to $] 0,1]$ ). 
Let us now handle the second term of the last sum we obtained. We have

$$
\int_{S_{\eta}}[x]_{\eta}^{\alpha-1}[x]_{\Delta \eta} \mathcal{H}^{1}(\mathrm{~d} x)=\int_{S_{\eta}} \mathcal{H}^{1}(\mathrm{~d} x) \int_{\Gamma} \Delta \eta(\mathrm{d} \gamma)[x]_{\eta}^{\alpha-1} I_{x \in \gamma} .
$$

Here we want to change the order of integration, and to do this we check what happens in absolute value:

$$
\begin{aligned}
\int_{S_{\eta}} \mathcal{H}^{1}(\mathrm{~d} x) \int_{\Gamma}|\Delta \eta|(\mathrm{d} \gamma)[x]_{\eta}^{\alpha-1} I_{x \in \gamma} & =\int_{\Gamma}|\Delta \eta|(\mathrm{d} \gamma) \int_{S_{\eta}} \mathcal{H}^{1}(\mathrm{~d} x)[x]_{\eta}^{\alpha-1} I_{x \in \gamma} \\
& =\int_{\Gamma}|\Delta \eta|(\mathrm{d} \gamma) \int_{0}^{\sigma(\gamma)}[\gamma(t)]_{\eta}^{\alpha-1} \mathrm{~d} t \\
& =\int_{\Gamma} Z_{\eta} \mathrm{d}|\Delta \eta|<+\infty .
\end{aligned}
$$

In this chain of equalities, the first one is just changing the integration order, while the second relies on the fact that $|\Delta \eta|$-a.e. we have $\mathcal{H}^{1}\left(\gamma \backslash S_{\eta}\right)=0$ and, $\gamma$ being parametrized by arc length, the $\mathcal{H}^{1}$-integral on its image becomes an integral with respect to $\mathrm{d} t$ on $[0, \sigma(\gamma)]$. The finiteness of the last integral in 3.3 allows us to change the order of integration with respect to $\Delta \eta$ and $\mathcal{H}^{1}$ and by analogous computations we get

$$
\int_{S_{\eta}} \mathcal{H}^{1}(\mathrm{~d} x) \int_{\Gamma} \Delta \eta(\mathrm{d} \gamma)[x]_{\eta}^{\alpha-1} I_{x \in \gamma}=\int_{\Gamma} Z_{\eta} \mathrm{d} \Delta \eta
$$

Inserting this last equality in 3.2 gives the conclusion.

\section{Existence of a landscape function and applications}

In this section we come specifically back to problem $(P)$ for $v=\delta_{0}$. Even when not explicitly stated, from now on $\eta$ will be an optimal pattern irrigating an $\alpha$-irrigable measure $\mu$.

\subsection{Well-definedness of the landscape function}

First a very elementary truncation lemma is needed. As it is just the formalization of a well-known principle (that a part of an optimal structure is itself optimal), it will not be proven here. It is in fact proven in [5] when stating the optimality of the connected components of a traffic plan in $\mathbb{R}^{d} \backslash\left\{x_{0}\right\}$.

LEMmA 4.1 If $\gamma_{0}$ is a curve such that $|\gamma|_{t_{0}, \eta}>0$, set $x_{0}=\gamma_{0}\left(t_{0}\right), A=\left[\gamma_{0}\right]_{t_{0}}, \mu_{A}=\left(\pi_{\infty}\right)_{\sharp}\left(I_{A} \cdot \eta\right)$, $\mu^{\prime}=\mu-\mu_{A}+\eta(A) \delta_{x_{0}}, \eta^{\prime}=\eta-I_{A} \cdot \eta+\eta(A) \delta_{\bar{\gamma}_{0}}$, where $\bar{\gamma}_{0}$ is the curve $\gamma_{0}$ stopped at time $t_{0}$. Then $\eta^{\prime}$ is an optimal pattern irrigating the measure $\mu^{\prime}$.

THEOREM 4.2 If $\gamma_{0}$ and $\gamma_{1}$ are two $\eta$-good curves sharing the same end-point $\bar{x}$, then $Z_{\eta}\left(\gamma_{0}\right)=$ $Z_{\eta}\left(\gamma_{1}\right)$.

Proof. If the two curves are identical the assertion is easy. If they are not identical, then they must split at a certain time $\bar{t}$. It is possible that one of them stops at time $\bar{t}$, but not both, as in this case they would be identical. So we can choose two times $t_{0}$ and $t_{1}$ with $\left|\gamma_{i}\right|_{i, \eta}>0$ and $\bar{t} \leqslant t_{i} \leqslant \sigma\left(\gamma_{i}\right)$ for $i=0,1$ (if one of the two curves stops at time $\bar{t}$, say for instance $\sigma\left(\gamma_{0}\right)=\bar{t}$, then we are 

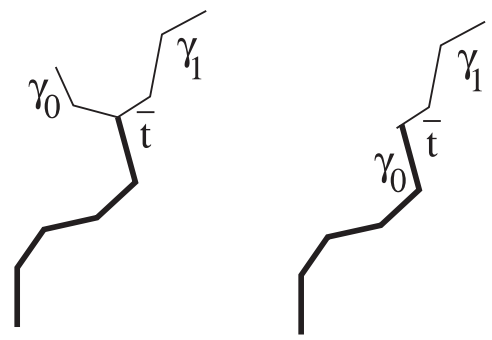

FIG. 3. Both curves may go on after $\bar{t}$ or one may stop.

forced to choose $t_{0}=\sigma\left(\gamma_{0}\right)=\bar{t}$ and we have $\left|\gamma_{0}\right|_{t_{0}, \eta}=\left|\gamma_{1}\right|_{\bar{t}, \eta}$ and $\left|\gamma_{1}\right|_{\bar{t}, \eta}>0$ as a consequence of $\left.\bar{t}<\sigma\left(\gamma_{1}\right)\right)$. Figure 3 shows the two possible situations.

Set $x_{i}=\gamma_{i}\left(t_{i}\right)$ and $l=\left|x_{1}-x_{0}\right|$. Then we use the notations of the previous lemma to write

$$
d_{\alpha}\left(\delta_{0}, \mu^{\prime}\right) \leqslant d_{\alpha}\left(\delta_{0}, \mu^{\prime \prime}\right)+d_{\alpha}\left(\mu^{\prime}, \mu^{\prime \prime}\right),
$$

where $\mu^{\prime \prime}=\mu-\mu_{A}+\eta(A) \delta_{x_{1}}$. Define $\eta^{\prime \prime}=\eta-I_{A} \cdot \eta+\eta(A) \delta_{\bar{\gamma}_{1}}$, where $\bar{\gamma}_{1}$ is $\gamma_{1}$ stopped at time $t_{1}$. It is easy to check that $\left(\pi_{\infty}\right)_{\sharp} \eta^{\prime \prime}=\mu^{\prime \prime}$ and then

$$
\begin{aligned}
d_{\alpha}\left(\delta_{0}, \mu^{\prime \prime}\right) & \leqslant J\left(\eta^{\prime \prime}\right) \leqslant J\left(\eta^{\prime}\right)+\alpha \int_{\Gamma} Z_{\eta} \mathrm{d}\left(\eta^{\prime \prime}-\eta^{\prime}\right) \\
& =J\left(\eta^{\prime}\right)+\alpha \eta(A)\left(\int_{0}^{t_{1}}\left|\gamma_{1}\right|_{t, \eta}^{\alpha-1} \mathrm{~d} t-\int_{0}^{t_{0}}\left|\gamma_{0}\right|_{t, \eta}^{\alpha-1} \mathrm{~d} t\right) .
\end{aligned}
$$

Here we have used Theorem 3.1 to estimate $J\left(\eta^{\prime \prime}\right)$. Actually by this theorem we would have $Z_{\eta^{\prime}}$ instead of $Z_{\eta}$. Yet we can replace $Z_{\eta^{\prime}}$ by $Z_{\eta}$ because we have only changed the measure $\eta$ on $A$ by the same amount of mass concentrated on $\bar{\gamma}_{0}$, and on $\gamma_{0} \cup \gamma_{1}$ this does not affect multiplicities. As far as the second term of the sum in (4.1) is concerned, it is easy to see that

$$
d_{\alpha}\left(\mu^{\prime}, \mu^{\prime \prime}\right) \leqslant \ln (A)^{\alpha} .
$$

By inserting these estimates in (4.1) we get

$$
\int_{0}^{t_{0}}\left|\gamma_{0}\right|^{\alpha-1} \mathrm{~d} t-\int_{0}^{t_{1}}\left|\gamma_{1}\right|^{\alpha-1} \mathrm{~d} t \leqslant \alpha^{-1} \ln (A)^{\alpha-1}
$$

Now estimate the length $l$ by

$$
\begin{aligned}
l & =\left|x_{0}-x_{1}\right| \leqslant\left|x_{0}-\bar{x}\right|+\left|\bar{x}-x_{1}\right| \leqslant\left(\sigma\left(\gamma_{0}\right)-t_{0}\right)+\left(\sigma\left(\gamma_{1}\right)-t_{1}\right) \\
& \leqslant \eta(A)^{1-\alpha} \int_{t_{0}}^{\sigma\left(\gamma_{0}\right)}\left|\gamma_{0}\right|_{t, \eta}^{\alpha-1} \mathrm{~d} t+\eta(B)^{1-\alpha} \int_{t_{1}}^{\sigma\left(\gamma_{1}\right)}\left|\gamma_{1}\right|_{t, \eta}^{\alpha-1} \mathrm{~d} t .
\end{aligned}
$$

Hence

$$
\int_{0}^{t_{0}}\left|\gamma_{0}\right|^{\alpha-1} \mathrm{~d} t-\int_{0}^{t_{1}}\left|\gamma_{1}\right|^{\alpha-1} \mathrm{~d} t \leqslant \alpha^{-1}\left(\int_{t_{0}}^{\sigma\left(\gamma_{0}\right)}\left|\gamma_{0}\right|_{t, \eta}^{\alpha-1} \mathrm{~d} t+\frac{\eta(B)^{1-\alpha}}{\eta(A)^{1-\alpha}} \int_{t_{1}}^{\sigma\left(\gamma_{1}\right)}\left|\gamma_{1}\right|_{t, \eta}^{\alpha-1} \mathrm{~d} t\right) .
$$


Notice that we cannot have $\left|\gamma_{i}\right|_{\sigma\left(\gamma_{i}\right), \eta}>0$ for both $i=0,1$, thanks to the no-loop property (property (3)). So, if $\left|\gamma_{1}\right|_{\sigma\left(\gamma_{1}\right), \eta}=0$, once we fix $t_{0}$ such that $\eta(A)>0$, we can choose $t_{1}$ so that $\eta(B) \leqslant \eta(A)$ since $\eta(B) \rightarrow 0$ as $t_{1} \rightarrow \sigma\left(\gamma_{1}\right)$. Otherwise, if $\left|\gamma_{1}\right|_{\sigma\left(\gamma_{1}\right), \eta}>0$, we can choose directly $t_{1}=\sigma\left(t_{1}\right)$. In both cases we have

$$
\int_{0}^{t_{0}}\left|\gamma_{0}\right|^{\alpha-1} \mathrm{~d} t-\int_{0}^{t_{1}}\left|\gamma_{1}\right|^{\alpha-1} \mathrm{~d} t \leqslant \alpha^{-1}\left(\int_{t_{0}}^{\sigma\left(\gamma_{0}\right)}\left|\gamma_{0}\right|_{t, \eta}^{\alpha-1} \mathrm{~d} t+\int_{t_{1}}^{\sigma\left(\gamma_{1}\right)}\left|\gamma_{1}\right|_{t, \eta}^{\alpha-1} \mathrm{~d} t\right) .
$$

Then we let $t_{0}$ and $t_{1}$ tend to $\sigma\left(\gamma_{0}\right)$ and $\sigma\left(\gamma_{1}\right)$, according to the criteria for the choice of $t_{1}$ we have used so far, and we get in the limit

$$
Z_{\eta}\left(\gamma_{0}\right)-Z_{\eta}\left(\gamma_{1}\right) \leqslant 0
$$

because the integrals on the right hand side of 4.2 tend to zero as a consequence of the fact that $\gamma_{0}$ and $\gamma_{1}$ are both $\eta$-good curves. Interchanging $\gamma_{0}$ and $\gamma_{1}$ proves the assertion.

COROLlaRY 4.3 If two different $\eta$-good curves $\gamma_{0}$ and $\gamma_{1}$ meet at a certain point $x=\gamma_{0}\left(t_{0}\right)=$ $\gamma_{1}\left(t_{1}\right)$, then $\left|\gamma_{0}\right|_{t_{0}, \eta}=\left|\gamma_{1}\right|_{t_{1}, \eta}=0$.

Proof. If one of the two multiplicities $\left|\gamma_{i}\right|_{t_{i}, \eta}$ were positive a strict inequality between $Z_{\eta}\left(\gamma_{0}\right)$ and $Z_{\eta}\left(\gamma_{1}\right)$ would hold, contrary to the equality just proven,

COROLlaRY 4.4 Any $\eta$-good curve $\gamma$ is in fact injective on $[0, \sigma(\gamma)]$.

Proof. The injectivity on $[0, \sigma(\gamma)$ [ is already known. Hence, consider the case $\gamma(\sigma(\gamma))=\gamma(t)$ for $t<\sigma(\gamma)$. This would imply $|\gamma|_{t, \eta}>0$, contrary to Corollary 4.3 applied to $\gamma$ and $\bar{\gamma}$, which is $\gamma$ stopped at time $t$.

REMARK 5 The injectivity on [0, $\sigma(\gamma)]$ was already known for $\eta$-a.e. curve $\gamma$ (see [4]). Yet, it was not possible to identify an explicit class of curves sharing this property. For the purposes of this paper it is important to switch from a generic "a.e." to the fact that this is true for $\eta$-good curves.

The result of Theorem 4.2 allows us to define a function on $\Omega$ via the values of $Z_{\eta}$.

DEFinition 4 We define the landscape function associated to the traffic plan $\eta$ as the function $z_{\eta}$ given by

$$
z_{\eta}(x)= \begin{cases}Z_{\eta}(\gamma) & \text { if } \gamma \text { is } \eta \text {-good and } x=\gamma(\sigma(\gamma)) \\ +\infty & \text { if no } \eta \text {-good curve ends at } x\end{cases}
$$

REMARK 6 It is in fact possible to prove more easily that the value of $z$ is well-defined $\mu$-a.e. (in the sense that if on a nonnegligible set of points $x$ we had two different values for $Z_{\eta}$ we would have the possibility to strictly improve the value of $J$ ). Yet, we do not want a function $z$ which is defined a.e. but a pointwise defined value, to deal later with pointwise properties, being also concerned with negligible sets such as $S_{\eta}$.

REMARK 7 Notice, as in Remark 4, that restrictions of $\eta$-good curves are still $\eta$-good and that this implies that if the landscape function is finite at a point $x$ then it is also finite on the whole $\eta$-good curve arriving at $x$. 
4.2 Variational applications: the functional $X_{\alpha}$

Some consequences of the existence of the landscape function are now presented.

COROLlaRY 4.5 For the functional $X_{\alpha}$ we have the following representation formula: $X_{\alpha}(\mu)=$ $\int_{\Omega} z \mathrm{~d} \mu$, where $z=z_{\eta}$ is the landscape function associated to any optimal pattern $\eta$ irrigating the measure $\mu$.

Proof. It is sufficient to apply the formula $X_{\alpha}(\mu)=J(\eta)=\int_{\Gamma} Z_{\eta} \mathrm{d} \eta$ and the fact that $Z_{\eta}(\gamma)$ depends only on $\pi_{\infty}(\gamma)$ through $Z_{\eta}(\gamma)=z\left(\pi_{\infty}(\gamma)\right)$ to get

$$
X_{\alpha}(\mu)=\int_{\Gamma} Z_{\eta} \mathrm{d} \eta=\int_{\Omega} z \mathrm{~d}\left(\left(\pi_{\infty}\right) \sharp \eta\right)=\int_{\Omega} z \mathrm{~d} \mu .
$$

COROLlary 4.6 If $\mu$ is $\alpha$-irrigable, then any landscape function $z$ is finite $\mu$-a.e.

Proof. Corollary 4.5 yields $\int z \mathrm{~d} \mu=X_{\alpha}(\mu)<+\infty$ and from this the result is straightforward.

REMARK 8 As the word "any" in the previous statement suggests, there is no uniqueness for the landscape function, and there is a landscape function for any optimal pattern.

Moreover, using Theorem 3.1 together with the existence of the landscape function, a derivation result extending the discrete case can be obtained. Notice that the following theorem will also be useful for other purposes, for instance when looking for continuity properties of the landscape function (see Section 6).

THEOREM 4.7 For a given function $g$ on $\Omega$ such that $\|g\|_{L^{\infty}(\mu)} \leqslant 1$ and such that $\int_{\Omega} g \mathrm{~d} \mu=0$, set $\mu_{1}=\mu(1+g)$. Then

$$
X_{\alpha}\left(\mu_{1}\right) \leqslant X_{\alpha}(\mu)+\alpha \int_{\Omega} z(x) g(x) \mu(\mathrm{d} x),
$$

where the function $z=z_{\eta}$ is the landscape function according to an arbitrary optimal pattern $\eta$ irrigating the measure $\mu$.

Proof. We will consider a variation of $\eta$ given by $\eta_{1}=(1+(g \circ \pi)) \cdot \eta$. Since $\pi_{\sharp} \eta_{1}=(1+g) \cdot \mu$, we have

$$
X_{\alpha}\left(\mu_{1}\right)-X_{\alpha}(\mu) \leqslant J\left(\eta_{1}\right)-J(\eta) .
$$

We want to apply Theorem 3.1 to this situation, with $\Delta \eta=(g \circ \pi) \cdot \eta$. Since $\Delta \eta$ is absolutely continuous with respect to $\eta$ with bounded density, it is straightforward that both the conditions required by the theorem ( $\Delta \eta$ being concentrated on $\Gamma_{\text {arc }} \cap \Gamma_{\text {inj }}$ and $Z_{\eta}$ being $|\Delta \eta|$-integrable) are satisfied, so

$$
J\left(\eta^{\prime}\right) \leqslant J(\eta)+\alpha \int_{\Gamma} Z_{\eta} \mathrm{d} \Delta \eta
$$

Now use the fact that $Z_{\eta}$ depends only on its terminal point to get

$$
\int_{\Gamma} Z_{\eta} \mathrm{d} \Delta \eta=\int_{\Omega} z \mathrm{~d}\left(\left(\pi_{\infty}\right)_{\sharp} \Delta \eta\right)=\int_{\Omega} z g \mathrm{~d} \mu .
$$

Putting together all the results yields the conclusion.

A simple consequence of this theorem may be expressed in terms of derivatives. 
COROLlaRY 4.8 Set $\mu_{\varepsilon}=\mu+\varepsilon g \cdot \mu$. Then the following derivative inequality holds:

$$
\limsup _{\varepsilon \rightarrow 0^{+}} \frac{X_{\alpha}(\mu+\varepsilon g \cdot \mu)-X_{\alpha}(\mu)}{\varepsilon} \leqslant \alpha \int_{\Omega} z(x) g(x) \mu(\mathrm{d} x) \text {. }
$$

\section{$4.3 \quad$ Variational applications: minimizing $\left(P_{+}\right)$}

As already mentioned, the last derivative inequality may be useful in variational problems involving $X_{\alpha}$. For the sake of clarity we provide a short example.

EXAMPLE 1 Consider the functional $F: \mathcal{P}(\Omega) \rightarrow[0,+\infty]$ given by

$$
F(\mu)= \begin{cases}\int_{\Omega} u^{2} \mathrm{~d} \mathcal{L}^{d} & \text { if } \mu=u \cdot \mathcal{L}^{d}, \\ +\infty & \text { if } \mu \text { is not absolutely continuous. }\end{cases}
$$

If we want to minimize (for $\alpha>1-1 / d$ ) the sum $X_{\alpha}(\mu)+F(\mu)$ over all probabilities $\mu$ on $\Omega$ we get as an optimality condition, by differentiating and using Corollary 4.8 .

$$
\alpha z+2 u=\text { const } \quad \text { a.e. on }\{u>0\} \text {. }
$$

This implies several interesting properties. First of all we notice that, both $z$ and $u$ being positive, they are also bounded. It was not $a$ priori evident that $u \in L^{\infty}(\Omega)$, since the natural condition was $u \in L^{2}(\Omega)$. Since $z(x) \geqslant|x|$, this also gives an estimate on the support of $\mu$. As the constant appearing in (4.3) could be uniformly estimated (it is sufficient to multiply (4.3) by $u$ and integrate, thus obtaining const $=\alpha X_{\alpha}(\mu)+2 F(\mu) \leqslant 2 \min \left(X_{\alpha}+F\right)$ ), this could also be used to prove an existence result for $\Omega=\mathbb{R}^{d}$, proving also that actually minimizers of $X_{\alpha}+F$ are supported in a given bounded ball. Moreover, formula (4.3) yields some regularity result for $u$ according to the results we will prove later on $z$.

Variational problems such as $\left(P_{+}\right)$have been first proposed in [15], where the authors suggested problems involving both the irrigation pattern $\chi$ and the irrigated measure $\mu_{\chi}$, and are very similar to those in [6]. In fact in [6] a similar sum is minimized, but with a standard Wasserstein distance instead of the term $X_{\alpha}(\mu)=d_{\alpha}\left(\mu, \delta_{0}\right)$. Such a model was proposed to study urban planning problems, with $\mu$ standing for the population density in a region, exactly as $\left(P_{+}\right)$may be used in studying the shape of a leaf or a flower, represented by $\mu$. In fact, the minimization of a sum of an $X_{\alpha}$ term and a convex functional on $\mu$ could be an easy model taking into account that leaves want to be as spread as possible to catch sunlight but have to be irrigated starting from a single source. In the framework of [6] the key condition coming from optimality was $\psi+f^{\prime}(u)=$ const, and the landscape function $z$ dealt with in this paper plays somehow the role of the Kantorovich potential $\psi$. Also Corollary 4.5 can be seen as indicating a similarity between the landscape function and the Kantorovich potential (see [19]). Moreover, the Hölder continuity result at the end of this paper perfectly agrees with the fact that Kantorovich potentials (which correspond to $\alpha=1$ ) are Lipschitz continuous.

\section{Properties of the landscape function}

\subsection{Semicontinuity}

LEMma 5.1 Given any $\eta \in \mathcal{P}(\Gamma)$, the function $Z_{\eta}: \Gamma \rightarrow \mathbb{R}$ is lower semicontinuous with respect to pointwise convergence. 
Proof. This result is almost implicitly proven both in [15] and in [4], but never explicitly stated. It is anyway proven that $x \mapsto[x]_{\eta}$ is upper semicontinuous, and hence $x \mapsto[x]_{\eta}^{\alpha-1}$ is 1.s.c. Then, to prove $\liminf _{n} Z_{\eta}\left(\gamma_{n}\right) \geqslant Z_{\eta}(\gamma)$, fix a time $t_{1}<\sigma(\gamma)$ and use $\liminf \sigma\left(\gamma_{n}\right) \geqslant \sigma(\gamma)$. Eventually we have $\sigma\left(\gamma_{n}\right)>t_{1}$ and, by Fatou's Lemma, we get

$$
\liminf _{n} Z_{\eta}\left(\gamma_{n}\right) \geqslant \liminf _{n} \int_{0}^{t_{1}}\left[\gamma_{n}\right]_{\eta}^{\alpha-1} \mathrm{~d} t \geqslant \int_{0}^{t_{1}}[\gamma]_{\eta}^{\alpha-1} \mathrm{~d} t
$$

Passing to the limit as $t_{1} \rightarrow \sigma(\gamma)$ gives the assertion.

THEOREM 5.2 The landscape function $z$ is lower semicontinuous.

Proof. Consider a sequence $x_{n} \rightarrow x$ and, correspondingly, some $\eta$-good curves $\gamma_{n}$ such that $\pi_{\infty}\left(\gamma_{n}\right)=x_{n}$ and $z\left(x_{n}\right)=Z_{\eta}\left(\gamma_{n}\right)$. We may assume $\sup _{n} z\left(x_{n}\right)<+\infty$. Since $\sigma\left(\gamma_{n}\right) \leqslant Z_{\eta}\left(\gamma_{n}\right)=$ $z\left(x_{n}\right)$, we also have $\sup _{n} \sigma\left(\gamma_{n}\right)<+\infty$ and we can extract a subsequence (not relabeled) such that $\gamma_{n} \rightarrow \gamma$ uniformly. It is not difficult to prove that $\pi_{\infty}(\gamma)=x$. Thus, it is sufficient to use Lemma 5.1 to get $Z_{\eta}(\gamma) \leqslant \liminf _{n} Z_{\eta}\left(\gamma_{n}\right)=\liminf _{n} z\left(x_{n}\right)$. This implies that $\gamma$ is an $\eta$-good curve and $z(x)=Z_{\eta}(\gamma)$, which yields the assertion.

\subsection{Maximal slope in the network direction}

The next property of the landscape function that can be proven in general (i.e., under no extra assumption on $\alpha, \Omega, \mu, \ldots)$ is most important in view of its meaning in river basin applications. Our interest is in a continuous counterpart of the landscape function of [2]. What we actually need is that, at the points of the irrigation network $S_{\eta}$, the direction of maximal slope of $z$ is exactly the direction of the network. If an $\eta$-good curve $\gamma_{0}$ is fixed, by the definition of $z$, for a.e. $t_{0}$ the derivative of $z$ along the curve $\gamma$ at the point $x_{0}=\gamma_{0}\left(t_{0}\right)$ is exactly $\left|\gamma_{0}\right|_{t_{0}, \eta}^{\alpha-1}$. This is why we prove the following result. Notice that, as already mentioned, in the continuous case the function $z$ cannot be expected to be very regular, and in fact the maximal slope result we are going to prove involves differentiability in a very pointwise way but very weak as well.

THEOREM 5.3 Let $x_{0}=\gamma_{0}\left(t_{0}\right)$, where $\gamma_{0}$ is an $\eta$-good curve, $t_{0}$ a time with $t_{0} \leqslant \sigma\left(\gamma_{0}\right)$ and $\theta_{0}:=\left|\gamma_{0}\right|_{t_{0}, \eta}>0$. Then, for any $x \notin \gamma_{0}\left(\left[0, t_{0}\right]\right)$,

$$
z(x) \geqslant z\left(x_{0}\right)-\theta_{0}^{\alpha-1}\left|x-x_{0}\right|-o\left(\left|x-x_{0}\right|\right) .
$$

This amounts to saying that the slope at $x_{0}$ in the direction of the network is actually the maximal slope at $x_{0}$.

Proof. Fix $x \notin \gamma_{0}\left(\left[0, t_{0}\right]\right)$ such that $z(x)<z\left(x_{0}\right)$. We may assume that $x=\gamma_{x}\left(t_{x}\right)$ for an $\eta$-good curve $\gamma_{x}$ (otherwise $z(x)=+\infty$ ) and that the two curves $\gamma_{0}$ and $\gamma_{x}$ get apart at a certain time $t_{1}(x)<t_{0}$ (the case $t_{1}(x) \geqslant t_{0}$ implies in fact $z(x) \geqslant z\left(x_{0}\right)$ ). By Lemma 5.4 below we know that $t_{1}(x) \rightarrow t_{0}$ as $\left|x-x_{0}\right| \rightarrow 0$. Set $\theta(t)=\left|\gamma_{0}\right|_{t, \eta}$; for $t \in\left[t_{1}(x), t_{0}\right]$ we may write $\theta(t) \leqslant \theta_{0}\left(1+\varepsilon_{x}\right)$, where $\varepsilon_{x}$ is infinitesimal as $\left|x-x_{0}\right| \rightarrow 0$ as a consequence of $t_{1}(x) \rightarrow t_{0}$.

We use again Lemma 4.1 and its notations. In particular $A=\left[\gamma_{0}\right]_{t_{0}}$ and $\theta_{0}=\theta\left(t_{0}\right)=\eta(A)$. Also define, as in Theorem 4.2. $\mu^{\prime \prime}=\mu-\mu_{A}+\eta(A) \delta_{x}$ and $\eta^{\prime \prime}=\eta-I_{A} \cdot \eta+\eta(A) \delta_{\bar{\gamma}_{x}}$, where $\bar{\gamma}_{x}$ is $\gamma_{x}$ stopped at time $t_{x}$, and it is easy to check that $\left(\pi_{\infty}\right)_{\sharp} \eta^{\prime \prime}=\mu^{\prime \prime}$. Then, by the optimality 


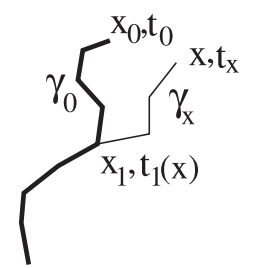

FIG. 4. Curves and points in the proof.

of $\eta^{\prime}$ (we recall that, according to the notations of Lemma 4.1 $\mu^{\prime}=\mu-\mu_{A}+\eta(A) \delta_{x_{0}}$ and $\eta^{\prime}=\eta-I_{A} \cdot \eta+\eta(A) \delta_{\bar{\gamma}_{0}}$, where $\bar{\gamma}_{0}$ is $\gamma_{0}$ stopped at $t_{0}$, see also Figure 44, we have

$$
J\left(\eta^{\prime}\right)=X_{\alpha}\left(\mu^{\prime}\right) \leqslant X_{\alpha}\left(\mu^{\prime \prime}\right)+d_{\alpha}\left(\mu^{\prime \prime}, \mu^{\prime}\right) \leqslant J\left(\eta^{\prime \prime}\right)+\left|x-x_{0}\right| \theta_{0}^{\alpha} .
$$

We want to compare $J\left(\eta^{\prime}\right)$ and $J\left(\eta^{\prime \prime}\right)$, and to do this we here need a more refined estimate than what we could find by using Theorem 3.1. As $\eta^{\prime}-\eta^{\prime \prime}=\theta_{0}\left(\delta_{\bar{\gamma}_{0}}-\delta_{\bar{\gamma}_{x}}\right)$, we have in particular $[y]_{\eta^{\prime \prime}}=[y]_{\eta^{\prime}}+\theta_{0}\left(I_{y \in \bar{\gamma}_{x}}-I_{y \in \bar{\gamma}_{0}}\right)$. By using 2.3$)$ we get

$$
J\left(\eta^{\prime \prime}\right)-J\left(\eta^{\prime}\right)=\int_{\bar{\gamma}_{x} \backslash \bar{\gamma}_{0}}\left(\left([y]_{\eta^{\prime}}+\theta_{0}\right)^{\alpha}-[y]_{\eta^{\prime}}^{\alpha}\right) \mathrm{d} \mathcal{H}^{1}-\int_{\bar{\gamma}_{0} \backslash \bar{\gamma}_{x}}\left([y]_{\eta^{\prime}}^{\alpha}-\left([y]_{\eta^{\prime}}-\theta_{0}\right)^{\alpha}\right) \mathrm{d} \mathcal{H}^{1} .
$$

It is not difficult to check that $[y]_{\eta^{\prime}}=[y]_{\eta}$ for $y \in \bar{\gamma}_{x} \cup \bar{\gamma}_{0}$, as we have replaced the part of $\eta$ concentrated on $A$ by an equal amount of mass on $\bar{\gamma}_{0}$. Hence we may estimate (rewriting the integrals with respect to $\mathcal{H}^{1}$ as integrals with respect to $\mathrm{d} t$ )

$$
J\left(\eta^{\prime \prime}\right)-J\left(\eta^{\prime}\right) \leqslant \alpha \int_{t_{1}(x)}^{t_{x}}\left|\gamma_{x}\right|_{t, \eta}^{\alpha-1} \theta_{0} \mathrm{~d} t-\int_{t_{1}(x)}^{t_{0}}\left(\theta(t)^{\alpha}-\left(\theta(t)-\theta_{0}\right)^{\alpha}\right) \mathrm{d} t .
$$

Since the function $s \mapsto s^{\alpha}-\left(s-\theta_{0}\right)^{\alpha}$ is decreasing and $\theta(t) \leqslant\left(1+\varepsilon_{x}\right) \theta_{0}$, we get $\theta(t)^{\alpha}-$ $\left(\theta(t)-\theta_{0}\right)^{\alpha} \geqslant \theta_{0}^{\alpha}\left(\left(1+\varepsilon_{x}\right)^{\alpha}-\varepsilon_{x}^{\alpha}\right)$. Hence

$$
J\left(\eta^{\prime \prime}\right)-J\left(\eta^{\prime}\right) \leqslant \alpha\left(z(x)-z\left(x_{1}\right)\right)-\left|t_{0}-t_{1}(x)\right| \theta_{0}^{\alpha}\left(\left(1+\varepsilon_{x}\right)^{\alpha}-\varepsilon_{x}^{\alpha}\right),
$$

where $x_{1}=\gamma_{0}\left(t_{1}(x)\right)=\gamma_{x}\left(t_{1}(x)\right)$. Write $\left(1+\varepsilon_{x}\right)^{\alpha}-\varepsilon_{x}^{\alpha}=\left(1+\varepsilon_{x}^{\prime}\right)^{-1}$ with $\varepsilon_{x}^{\prime}>0$ infinitesimal as $x \rightarrow x_{0}$. From $\theta_{0}^{\alpha-1} \geqslant(\theta(t))^{\alpha-1}$ we get $\left|t_{0}-t_{1}(x)\right| \theta_{0}^{\alpha} \geqslant \theta_{0}\left(z\left(x_{0}\right)-z\left(x_{1}\right)\right)$. Now notice that, for $\left|x-x_{0}\right|$ sufficiently small, the inequality $\alpha<\left(1+\varepsilon_{x}^{\prime}\right)^{-1}$ is satisfied, and hence

$$
J\left(\eta^{\prime \prime}\right) \leqslant J\left(\eta^{\prime}\right)+\left(1+\varepsilon_{x}^{\prime}\right)^{-1} \theta_{0}\left(z(x)-z\left(x_{0}\right) .\right.
$$

If we insert this into (5.1) we finally get

$$
z(x)-z\left(x_{0}\right) \geqslant-\theta_{0}^{\alpha-1}\left|x-x_{0}\right|\left(1+\varepsilon_{x}^{\prime}\right) .
$$

LEMma 5.4 According to the notations of Theorem 5.3 , when $x \rightarrow x_{0}$ and $z(x) \leqslant z\left(x_{0}\right)$, the parting time $t_{1}(x)$ tends to $t_{0}$.

Proof. Suppose, by contradiction, that there exists a sequence $x_{k} \rightarrow x_{0}$ such that $\lim _{k} t_{1}\left(x_{k}\right)=$ $\bar{t}<t_{0}$ and $z\left(x_{k}\right) \leqslant z\left(x_{0}\right)$. Since $\gamma_{0}$ is injective (Corollary 4.4), we may infer the existence of a 
positive quantity $\delta$ such that $\left|\gamma_{0}\left(t_{1}\left(x_{k}\right)\right)-x_{0}\right| \geqslant \delta$ (otherwise there would be a time $t \leqslant \bar{t}<t_{0}$ with $\left.\gamma_{0}(t)=x_{0}\right)$. For any $k$ consider an $\eta$-good curve $\gamma_{k}$ such that $x_{k}=\gamma_{k}\left(t_{k}\right)$. First notice that, at least for $k$ large enough, thanks to $\left|\gamma_{k}\left(t_{1}\left(x_{k}\right)\right)-x_{k}\right|=\left|\gamma_{0}\left(t_{1}\left(x_{k}\right)\right)-x_{k}\right| \rightarrow\left|\gamma_{0}(\bar{t})-x_{0}\right| \geqslant \delta$, we have $t_{k}>\bar{t}+\delta / 2$. Then consider the points $\gamma_{k}(\bar{t}+\delta / 2)$ : this collection of points must in fact be finite, otherwise we would have $\left|\gamma_{k}\right|_{\bar{t}+\delta / 2, \eta} \rightarrow 0$ and hence $z\left(x_{k}\right) \geqslant\left|\gamma_{k}\right|_{\bar{t}+\delta / 2, \eta}^{\alpha-1}\left|t_{k}-(\bar{t}+\delta / 2)\right| \rightarrow+\infty$ because $\left|t_{k}-(\bar{t}+\delta / 2)\right| \geqslant\left|x_{k}-\gamma_{0}(\bar{t})\right|-\delta / 2 \geqslant \delta / 2$. This contradicts $z\left(x_{k}\right) \leqslant z\left(x_{0}\right)$ and so we may suppose, up to subsequences, that $\gamma_{k}(\bar{t}+\delta / 2)=\bar{x}$ (for a point $\bar{x}$ which does not belong to the image of $\gamma_{0}$, otherwise we would contradict property (3)) and that $\gamma_{k}$ uniformly converges to a curve $\gamma$. In the limit we should get a curve $\gamma$ passing through $\gamma_{0}(\bar{t}), \bar{x}$ and $x_{0}$, i.e. we have created a loop because $\gamma_{0}$ does not pass through $\bar{x}$ (see Figure also 5 .

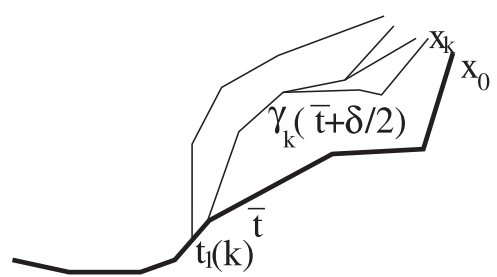

FIG. 5. A sequence of curves creating a loop in the limit.

From $z\left(x_{k}\right) \leqslant z\left(x_{0}\right)$ we can infer by semicontinuity (Lemma 5.1) that $\gamma$ is an $\eta$-good curve and hence this loop contradicts Corollary 4.3 .

\section{Hölder continuity under extra assumptions}

Here we will be able to prove some extra regularity properties of $z$, but we have to add some assumptions. The most important ones are on $\alpha(\alpha>1-1 / d$ is required) and on the irrigated measure $\mu$ (a lower bound on its density is supposed).

\subsection{Campanato spaces by medians}

We will here give a simple variant of a well-known result by Campanato (see [7]) about an integral characterization of Hölder continuous functions.

Definition 5 Given a measurable function $u$ on a domain $U$ we define a median of $u$ in $U$ to be any number $m$ which satisfies the following equivalent conditions:

- $|\{x \in U: u(x)>m\}| \leqslant \frac{1}{2}|U|$ and $|\{x \in U: u(x)<m\}| \leqslant \frac{1}{2}|U|$;

- there exists a measurable subset $A \subset\{x \in U: u(x)=m\}$ such that $|\{x \in U: u(x)>m\} \cup A|=$ $\frac{1}{2}|U|$

- the function $t \mapsto \int_{U}|u(x)-t| \mathrm{d} x$ achieves its minimum at $t=m$.

The set of medians of $u$ in $U$ is an interval of $\mathbb{R}$; the middle point of this interval is called the central median of $u$ in $U$.

Definition 6 If $A$ is a given positive number, a domain $\Omega \subset \mathbb{R}^{d}$ is said to be of type $A$ if $\left|\Omega_{x_{0}, r}\right| \geqslant A r^{d}$ for any $x_{0} \in \Omega$ and $r \in[0, \operatorname{diam} \Omega]$, where $\Omega_{x_{0}, r}=\Omega \cap B\left(x_{0}, r\right)$. 
LEMMA 6.1 If $\Omega$ is a domain of type $A$ and $u$ is a function in $L^{1}(\Omega)$ such that

$$
\int_{\Omega_{x_{0}, r}}\left|u-\tilde{u}_{x_{0}, r}\right| \mathrm{d} x \leqslant C r^{d+\beta}
$$

for a finite constant $C$ and any $r \in[0, \operatorname{diam} \Omega]$, where $\tilde{u}_{x_{0}, r}$ is the central median of $u$ on $\Omega_{x_{0}, r}$, then $u$ admits a representative which is Hölder continuous with exponent $\beta$.

Proof. This is nothing but the fact that Campanato spaces may be built by using medians instead of average values (adapt the proof of Theorem 1.2, page 70 in [10]). In fact, it is easy to see that for each point $x_{0}$ the value $\tilde{u}_{x_{0}, r}$ converges as $r \rightarrow 0$ to a value $\tilde{u}\left(x_{0}\right)$ and that

$$
|\tilde{u}(x)-\tilde{u}(y)| \leqslant C|x-y|^{\beta},
$$

exactly as in the proof we mentioned. What we need is that $\tilde{u}(x)=u(x)$ a.e. This can be shown in this way: denote the average value of $u$ on $\Omega_{x_{0}, r}$ by $\bar{u}_{x_{0}, r}$. Then

$$
\left|\bar{u}_{x_{0}, r}-\tilde{u}_{x_{0}, r}\right| \leqslant\left|\Omega_{x_{0}, r}\right|^{-1} \int_{\Omega_{x_{0}, r}}\left|u(x)-\tilde{u}_{x_{0}, r}\right| \mathrm{d} x \leqslant\left|\Omega_{x_{0}, r}\right|^{-1} \int_{\Omega_{x_{0}, r}}\left|u(x)-\bar{u}_{x_{0}, r}\right| \mathrm{d} x,
$$

where the second inequality is a consequence of the minimality property of the median. As at Lebesgue points the last expression tends to zero, this implies that the average $\bar{u}_{x_{0}, r}$ and the median $\tilde{u}_{x_{0}, r}$ share the same limit a.e. At the same points we also have $\bar{u}_{x_{0}, r} \rightarrow u\left(x_{0}\right)$, and this proves $\tilde{u}\left(x_{0}\right)=u\left(x_{0}\right)$ a.e.

\subsection{Hölder continuity of the landscape function}

THEOREM 6.2 Suppose that $\Omega$ is a domain of type $A$ for $A>0$, that $\alpha>1-1 / d$ and that $\mu \in \mathcal{P}(\Omega)$ is a probability measure such that the density of its absolutely continuous part is bounded from below by a positive constant. Then any landscape function $z$ has a representative $\tilde{z}$ which is Hölder continuous with exponent $\beta=d(\alpha-(1-1 / d))$.

Proof. Fix a measure $\mu_{1}$ and apply Theorem 4.7 to it and $\mu$. By using the triangle inequality for $d_{\alpha}$, we get

$$
-d_{\alpha}\left(\mu, \mu_{1}\right) \leqslant X_{\alpha}\left(\mu_{1}\right)-X_{\alpha}(\mu) \leqslant \alpha \int_{\Omega} z \mathrm{~d}\left(\mu_{1}-\mu\right),
$$

provided $\mu_{1}$ is a measure of the form allowed in Theorem 4.7 , i.e. $\mu_{1} \ll \mu$ with bounded density. From 6.1 we get

$$
\alpha \int_{\Omega} z \mathrm{~d}\left(\mu-\mu_{1}\right) \leqslant d_{\alpha}\left(\mu, \mu_{1}\right)
$$

Suppose that $\mu$ has an absolutely continuous part with density everywhere larger than $\lambda_{0}>0$ and choose

$$
\mu_{1}=\mu-\lambda_{0} I_{A} \cdot \mathcal{L}^{d}+\lambda_{0} I_{B} \cdot \mathcal{L}^{d},
$$

where $A$ and $B$ are measurable subsets of $\Omega_{x_{0}, \varepsilon}$ with $|A|=|B|, A \cup B=\Omega_{x_{0}, \varepsilon}$ and $A \subset\{z \geqslant m\}$ and $B \subset\{z \leqslant m\}$ and $m$ is the central median value for $z$ in $\Omega_{x_{0}, \varepsilon}$. By construction $\mu_{1}$ is a 
probability measure to which the estimate of Theorem 4.7 may be applied. With this choice of $\mu$ and $\mu_{1}$ we get

$$
\begin{aligned}
\int_{\Omega} z \mathrm{~d}\left(\mu-\mu_{1}\right) & =\int_{A} z(x) \lambda_{0} \mathrm{~d} x-\int_{B} z(x) \lambda_{0} \mathrm{~d} x \\
& =\lambda_{0}\left(\int_{A}(z(x)-m) \mathrm{d} x-\int_{B}(z(x)-m) \mathrm{d} x\right)=\lambda_{0} \int_{\Omega_{x_{0}, \varepsilon}}|z(x)-m| \mathrm{d} x .
\end{aligned}
$$

Putting this into 6.2 yields

$$
\int_{\Omega_{x_{0}, \varepsilon}}|z(x)-m| \mathrm{d} x \leqslant\left(\alpha \lambda_{0}\right)^{-1} d_{\alpha}\left(\mu, \mu_{1}\right) .
$$

To estimate $d_{\alpha}\left(\mu, \mu_{1}\right)$ use 2.1 to get

$$
\int_{\Omega_{x_{0}, \varepsilon}}|z(x)-m| \mathrm{d} x \leqslant \frac{C_{\alpha, d}}{\lambda_{0}^{1-\alpha}} \varepsilon^{1+\alpha d} .
$$

Since $1+\alpha d=d+\beta$, Lemma 6.1 may be applied.

An important consequence is the following:

COROLLARY 6.3 Under the same assumptions on $\Omega, \alpha$ and $\mu$ of Theorem 6.2

$$
X_{\alpha}\left(\mu_{1}\right) \leqslant X_{\alpha}(\mu)+\int_{\Omega} \tilde{z} \mathrm{~d}\left(\mu_{1}-\mu\right) \quad \text { for any measure } \mu_{1} \in \mathcal{P}(\Omega) .
$$

Proof. The inequality holds for $\mu_{1}$ of the form $\mu_{1}=(1+g) \cdot \mu$ with $g \in L^{\infty}$, but any measure $\mu_{1} \in \mathcal{P}(\Omega)$ may be approximated by such measures. Since $\tilde{z}$ is continuous, on both sides of the inequalities we have quantities which are continuous in $\mu_{1}$ with respect to weak convergence. This shows that the same inequality is valid for any $\mu_{1}$.

Even if we have proven that the landscape function $z$ equals a.e. a function which is Hölder continuous, this is not enough. In fact, this does not provide information on the behavior of $z$ on negligible sets. Yet, the pointwise values of $z$ on $S_{\eta}$ are of particular interest (as in the last section), and $S_{\eta}$ is one-dimensional and thus negligible. This is why the next step will be proving that $z$ and $\tilde{z}$ actually agree everywhere.

THEOREM 6.4 Let $m_{\varepsilon}$ denote the central median of $z$ in the ball $B\left(x_{0}, \varepsilon\right)$. Under the same assumptions of Theorem 6.2 one has $m_{\varepsilon} \rightarrow z\left(x_{0}\right)$ as $\varepsilon \rightarrow 0$. Consequently, $\tilde{z}\left(x_{0}\right)=z\left(x_{0}\right)$.

Proof. By the semicontinuity of $z$ it is easy to get $\liminf _{\varepsilon \rightarrow 0} m_{\varepsilon} \geqslant z\left(x_{0}\right)$, hence only an estimate from above for $m_{\varepsilon}$ is needed. Consider a ball $B\left(x_{0}, \varepsilon\right)$ and a set $A_{\varepsilon} \subset B\left(x_{0}, \varepsilon\right) \cap\left\{z \geqslant m_{\varepsilon}\right\}$ such that $\left|A_{\varepsilon}\right|=\left|B\left(x_{0}, \varepsilon\right)\right| / 2$. Then set $\Gamma_{\varepsilon}=\left\{\gamma \in \Gamma:\left(\pi_{\infty}\right)(\gamma) \in A_{\varepsilon}\right\}, \mu_{\varepsilon}=\mu+\mu\left(A_{\varepsilon}\right) \delta_{x_{0}}-I_{A_{\varepsilon}} \cdot \mu$, and $\eta_{\varepsilon}=\eta+\eta\left(\Gamma_{\varepsilon}\right) \delta_{\gamma_{0}}-I_{\Gamma_{\varepsilon}} \cdot \eta$, where $\gamma_{0}$ is an $\eta$-good curve stopping at $x_{0}$. Theorem 3.1 can be applied to $\eta$ and $\eta_{\varepsilon}$ and hence

$$
\begin{aligned}
J\left(\eta_{\varepsilon}\right) & \leqslant J(\eta)+\alpha\left(\eta\left(\Gamma_{\varepsilon}\right) Z_{\eta}\left(\gamma_{0}\right)-\int_{\Gamma_{\varepsilon}} Z_{\eta} \mathrm{d} \eta\right) \\
& =J(\eta)+\alpha \mu\left(A_{\varepsilon}\right) z\left(x_{0}\right)-\alpha \int_{A_{\varepsilon}} z(x) \mu(\mathrm{d} x) \leqslant J(\eta)+\alpha \mu\left(A_{\varepsilon}\right)\left(z\left(x_{0}\right)-m_{\varepsilon}\right) .
\end{aligned}
$$


It follows that

$$
X_{\alpha}(\mu) \leqslant X_{\alpha}\left(\mu_{\varepsilon}\right)+C \varepsilon \mu\left(A_{\varepsilon}\right)^{\alpha} \leqslant X_{\alpha}(\mu)+\alpha \mu\left(A_{\varepsilon}\right)\left(z\left(x_{0}\right)-m_{\varepsilon}\right)+C \varepsilon \mu\left(A_{\varepsilon}\right)^{\alpha} .
$$

This implies

$$
m_{\varepsilon}-z\left(x_{0}\right) \leqslant C \varepsilon \mu\left(A_{\varepsilon}\right)^{\alpha-1} \leqslant C \varepsilon^{1+d(\alpha-1)} .
$$

Since $1+d(\alpha-1)>0$ we get $\limsup _{\varepsilon \rightarrow 0} m_{\varepsilon} \leqslant z\left(x_{0}\right)$. To get the second assertion, just use $\tilde{z}\left(x_{0}\right)=\lim _{\varepsilon \rightarrow 0} m_{\varepsilon}$.

REMARK 9 The landscape function $z$ is in general never Lipschitz continuous (not even locally), as on the set $S_{\eta}$ it has slopes given by $\theta^{\alpha-1}$. This means that, if we have arbitrarily small values of $\theta$, we cannot have a Lipschitz constant for $z$. Yet estimates of the kind $\theta \geqslant c>0$ would imply $\mathcal{H}^{1}\left(S_{\eta}\right)<+\infty$ and no measure whose support is not one-dimensional may be irrigated by a set of finite length (or locally finite length).

\section{Acknowledgments}

The work has been completed during a research stay at CMLA-Cachan, financed by an international grant by ENS Cachan, which is gratefully acknowledged. On this occasion, Prof. Morel read a previous version of the paper maybe more carefully than the author himself and suggested lots of improvements. For his help, suggestions and support the author wants to thank him very much. As another personal remark, it has been a pleasure to cite and use results on Campanato spaces, as the late Prof. Campanato had been the author's first calculus teacher, in his last teaching year in Pisa.

\section{REFERENCES}

1. Banavar, J. R., Colaiori, F., Flammini, A., Giacometti, A., Maritan, A., \& Rinaldo, A. Sculpting of a fractal river basin. Phys. Rev. Lett. 78 (1997), 4522-4525.

2. Banavar, J. R., Colaiori, F., Flammini, A., Maritan, A., \& Rinaldo, A. Scaling, optimality, and landscape evolution. J. Statist. Phys. 104 (2001), 1-48. Zbl 1074.86518

3. Bernot, M. Optimal transport and irrigation. PhD Thesis, ENS Cachan (2005), http://perso.crans. org/ bernot.

4. Bernot, M., Caselles, V., \& Morel, J.-M. Traffic plans. Publ. Mat. 49 (2005), 417-451. Zbl pre02233222 MR 2177636

5. Bernot, M., Caselles, V., \& Morel, J.-M. The structure of branched transportation networks. Calc. Var. Partial Differential Equations, to appear; http://www.cmla.ens-cachan.fr/Cmla/.

6. Buttazzo, G., \& Santambrogio, F. A model for the optimal planning of an urban area. SiAM J. Math. Anal. 37 (2005), 514-530. Zbl pre05029345 MR 2176114

7. Campanato, S. Proprietà di Hölderianità di alcune classi di funzioni. Ann. Scuola Norm. Sup. Pisa 17 (1963), 175-188. Zbl 0121.29201 MR 0156188

8. Devillanova, G. Singular structures in some variational problems. PhD Thesis, ENS Cachan (2005).

9. Devillanova, G., \& Solimini, S. Elementary properties of optimal irrigation patterns. Calc. Var. Partial Differential Equations, to appear.

10. Giaquinta, M. Multiple Integrals in the Calculus of Variations and Nonlinear Elliptic Systems, Princeton Univ. Press (1983). Zbl 0516.49003 MR 0717034

11. GiLbert, E. N. Minimum cost communication networks. Bell System Tech. J. 46 (1967), 2209-2227. 
12. Gilbert, E. N., \& Pollak, H. O. Steiner minimal trees. SiAM J. Appl. Math. 16 (1968), 1-29. Zbl 0159.22001 MR 0223269

13. IjJasz-Vasquez, E., Bras, R. L., Rodriguez-Iturbe, I., Rigon, R., \& Rinaldo, A. Are river basins optimal channel networks? Adv. Water Resources 16 (1993), 69-79.

14. Maddalena, F., \& Solimini, S. Transport distances and irrigation models. Preprint, http:// cvgmt.sns.it/papers/madsol07/.

15. Maddalena, F., Solimini, S., \& Morel, J.-M. A variational model of irrigation patterns. Interfaces Free Bound. 5 (2003), 391-415. Zbl 1057.35076 MR 2031464

16. Rigon, R., Rinaldo, A., Rodriguez-Iturbe, I., IjJasz-VAsquez, E., \& Bras, R. L. Optimal channel networks: a framework for the study of river basin morphology. Water Resources Res. 29 (1993), $1635-1646$.

17. Rodriguez-Iturbe, I., \& Rinaldo, A. Fractal River Basins. Cambridge Univ. Press (1997).

18. Rodriguez-Iturbe, I., Rinaldo, A., Rigon, R., BRAs, R. L., IJjasz-VAsquez, E., \& MARAni, A. Fractal structures as least energy dissipation patterns: the case of river networks. Geophys. Res. Lett. 5 (1992), 2854-2860.

19. Villani, C. Topics in Optimal Transportation. Grad. Stud. Math. 58, Amer. Math. Soc. (2003).

20. XIA, Q. Optimal paths related to transport problems. Commun. Contemp. Math. 5 (2003), 251-279. Zbl 1032.90003 MR 1966259

21. XIA, Q. Interior regularity of optimal transport paths. Calc. Var. Partial Differential Equations 20 (2004), 283-299. Zbl 1080.90008 MR 2062945 